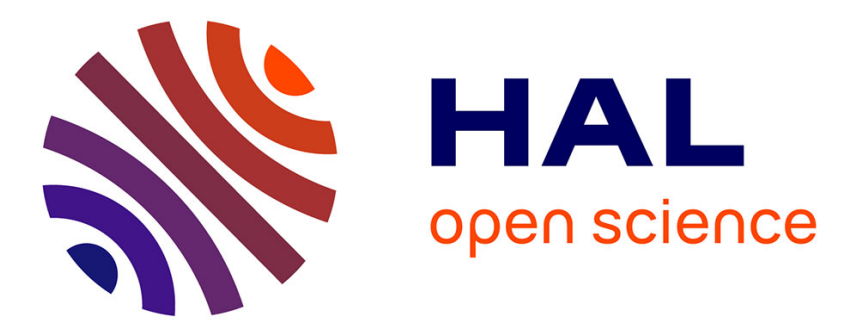

\title{
Numerical study on Kerr frequency comb generation in Si 3 N 4 microresonators with frequency-dependent access coupler properties
}

Napoléon Gutierrez, Arnaud Fernandez, Olivier Llopis, Stéphane Calvez, Stéphane Balac

\section{To cite this version:}

Napoléon Gutierrez, Arnaud Fernandez, Olivier Llopis, Stéphane Calvez, Stéphane Balac. Numerical study on Kerr frequency comb generation in Si $3 \mathrm{~N} 4$ microresonators with frequency-dependent access coupler properties. Journal of the Optical Society of America B, 2019, 36 (10), pp.2896-2906. 10.1364/JOSAB.36.002896 . hal-02298739

\section{HAL Id: hal-02298739 \\ https://hal.science/hal-02298739}

Submitted on 27 Sep 2019

HAL is a multi-disciplinary open access archive for the deposit and dissemination of scientific research documents, whether they are published or not. The documents may come from teaching and research institutions in France or abroad, or from public or private research centers.
L'archive ouverte pluridisciplinaire HAL, est destinée au dépôt et à la diffusion de documents scientifiques de niveau recherche, publiés ou non, émanant des établissements d'enseignement et de recherche français ou étrangers, des laboratoires publics ou privés. 


\title{
Numerical study on Kerr frequency comb generation in $\mathrm{Si}_{3} \mathrm{~N}_{4}$ microresonators with frequency-dependent access coupler properties
}

\author{
Napoleon Gutierrez, ${ }^{1}$ Arnaud Fernandez, ${ }^{1,}{ }^{*}$ Olivier Llopis, ${ }^{1}$ Stephane \\ CAlvez, ${ }^{1}$ Stephane Balac ${ }^{2}$ \\ ${ }^{1}$ LAAS-CNRS, Université de Toulouse, CNRS, UPS, Toulouse, France \\ ${ }^{2}$ UNIV Rennes, CNRS, IRMAR-UMR 6625, F-35000 Rennes, France \\ *Corresponding author: afernand@laas.fr
}

Received XX Month XXXX; revised XX Month, XXXX; accepted XX Month XXXX; posted XX Month XXXX (Doc. ID XXXXX); published XX Month XXXX

Within the frame of vertically coupled silicon nitride $\left(\mathrm{Si}_{3} \mathrm{~N}_{4}\right)$ resonators characterized with $220 \mathrm{GHz}$ free spectral range driven by a continuous wave laser at $1.55 \mu \mathrm{m}$ for Kerr comb generation, the design of achromatic critically coupled resonators may bring severe issues because of the difficulty in designing dispersion-free access couplers with controlled coupling factor over a large spectral bandwidth. As a consequence of this, numerical simulations of Kerr frequency comb in these structures may drastically differ from reality if frequency-dependence of the access coupler's properties is not taken into account in the simulated model. In order to address this issue, we have developed a numerical model that takes into account the frequency dependence of the access coupler coefficients and remains valid even for the simulation of low $Q$ factor resonators. Field propagation within the ring is described by the nonlinear Schrödinger equation. Novelty in the model resides in the computation of a complex-valued, frequency dependent coupling transfer function between resonant ring and underlying access waveguide that models frequency-dependent dispersion and losses in the access coupling region of the resonator. Based on simulation results, we discuss on the differences observed in Kerr comb generation in resonators with three different coupler designs initially intended to yield critical coupling over the largest achievable bandwidth.

http://dx.doi.org/10.1364/AO.99.099999

\section{Introduction}

The work of T. Hänsch and J. Hall on optical frequency combs with mode-locked lasers has contributed to optical frequency metrology by enabling a coherent link between optical and microwave frequencies [1]. This work has allowed and led to the development of new technologies and applications like coherent terabit telecommunication [2], ultra-short pulse generation [3], optical waveform synthesis [4], astronomical spectrometry [5], comb spectroscopy [6], low phase noise microwave and terahertz generation [7].

Besides the generation techniques based on femtosecond mode locked lasers [8], optical resonators present an attractive and compact alternative to these systems due to their mode selectivity and power enhancement properties. The high quality factor (Q) that is achievable with optical resonators along with the strong confinement of light in the mode volume of the optical medium that makes up the microresonators are very favorable properties for the generation of Kerr Frequency combs (KFC) with low pump power threshold. Since Q factor is ultimately determined by the material absorption, the highest
$\mathrm{Q}$ factors are achievable with fused silica micro sphere [9], $\mathrm{CaF}_{2}$ [10], $\mathrm{MgF}_{2}$ [3] and $\mathrm{SrF}_{2}$ [11] toroidal resonators, materials that allow $\mathrm{Q}$ factor values higher than $10^{10}$. However, achieving ultra-high total $Q$ factor requires precise control of the coupling system which is delicate with tapered fibers and technically challenging with prism injection [12]. Despite a higher absorption, silicon nitride $\left(\mathrm{Si}_{3} \mathrm{~N}_{4}\right)$ planar resonators offer an interesting alternative due to higher nonlinear refractive index $\left(n_{2}=2.510^{-15} \mathrm{~cm}^{2} . W^{-1}\right)$ of the material which compensates lower $\mathrm{Q}$ factors that usually do not exceed $10^{7} \mathrm{in}$ these structures. On top of that, this technology is robust, compatible with CMOS technology and operational in the telecommunication band free of two photon absorption and free carrier absorption [13]. It has been proven that dispersion engineering in these structures can be adjusted to provide adequate dispersive properties for KFC generation at almost any wavelength [14]. In addition to this, the generation of an octave spanning KFC covering $128 \mathrm{THz}$ from a $\mathrm{Si}_{3} \mathrm{~N}_{4}$ resonator with $226 \mathrm{GHz}$ FSR has already been experimentally demonstrated by Okawachi etal. [15].

In the last decade, intensive numerical studies on KFC generation have been published [16-20]. The main formalisms used to model 
nonlinear optical cavities are the coupled mode equations and LugiatoLefever (LL) formalism. These formalisms model the dynamics of high finesse optical cavities and rely on the hypothesis that variations of the intra-cavity field between successive round-trips is small [21,22]. Coupled mode equations, where intra-cavity comb dynamics are described by a set of coupled partial differential equations that each describe the dynamics of each resonator mode is an advantageous modelling approach that allows for the implementation of frequency dependent losses, coupling coefficients and Kerr nonlinear coefficient [18] but remains valid for high finesse cavities only. A variety of numerical methods that each presents different advantages have been put in place to solve the LL equation. Common approaches to solve the LL equation and study the dynamics of nonlinear optical cavities are split-step based methods. Note that also a multidimensional root-finding Newton-Raphson method has been used in [16] to very quickly obtain steady-state solutions of the LL equation.

In the case of vertically coupled $\mathrm{Si}_{3} \mathrm{~N}_{4} / \mathrm{SiO}_{2}$ racetrack resonators (Fig. 1), previous work led by Arlotti [23] has demonstrated a significant frequency dependence of complex-valued coupling coefficients that end up inducing additional fluctuations of $\mathrm{Q}$ factor and dispersion with frequency. Taking into account this frequencydependent coupling, strong and potentially significant degradations of resonator quality factor become apparent which may impact KFC formation dynamics and shape. With lower $\mathrm{Q}$ factor, intra-cavity field variations between successive round-trips may become more pronounced and thus conventional modelling approaches may not be best suited to approach these specific cases of study.

In this this paper we propose a numerical iterative scheme based on the Ikeda map [24] that separates field propagation in the ring resonator from optical coupling between the ring and the access waveguide similar to the one used in reference [25]. The Ikeda map model remains valid even when strong fluctuations of intra-cavity field between successive round-trips take place. Furthermore, because coupling and intra-cavity propagation are each treated separately, this numerical scheme easily allows the implementation of non-reciprocal and frequency-dependent access coupler transfer function of a resonator while correctly modelling the behavior of the device. This is, however, at the expense of higher computation cost compared to the LL equation since the propagation step-size cannot be larger than the cavity length when using this model as the basis for simulations. A fourth order, Runge-Kutta method in the interaction picture (RK4IP) is used to solve the nonlinear Schrödinger equation over the length of the cavity and simulate intra-cavity field propagation [26].

All along this paper we will compare three specific designs of vertically coupled $\mathrm{Si}_{3} \mathrm{~N}_{4} / \mathrm{SiO}_{2}$ resonators with the same resonant ring but different coupling designs that were theoretically studied in [23] in order to obtain critical coupling over a spectral range larger than $30 \mathrm{~nm}$ at $1550 \mathrm{~nm}$ pump wavelength. Section 1 of this paper is dedicated to the description of the numerical model. In section 2 , the cold cavity transfer function properties are described. The main numerical results on Kerr frequency comb generation are presented and discussed in section 3 .

\section{Description of the lkeda map for KFC}

The approach presented in this paper consists in modelling the cavity by the means of an iterative method that computes separately the propagation of the electrical field within the ring resonator from the coupling of an external, initially blue detuned, cw pump laser whose central frequency is linearly shifted toward lower frequencies across the cold resonator resonance frequency. This iterative approach combines at each iteration two models: the Generalized NonLinear Schrödinger Equation (GNLSE) that describes intra-cavity light propagation and a transfer matrix model that describes propagation through the coupler. The use of the Ikeda map to model the optical cavity allows an interesting degree of latitude as it can be applied to any optical cavity regardless of its finesse or quality factor, thus providing a reliable tool for the implementation of the information about frequency-dependent coupling provided by Arlotti et al. in [23] in the simulation of $\mathrm{KFC}$ generation.

In Fig. 1, the ring resonator schematic representation depicts a ring resonator with cavity length $L$ corresponding to a free spectral range $F S R=c / n_{g} L$ where $n_{g}$ is the ring resonator group index at pump wavelength and $c$ the speed of light in vacuum. The round-trip time of the pump laser frequency is given by $T_{R}=1 / F S R$.

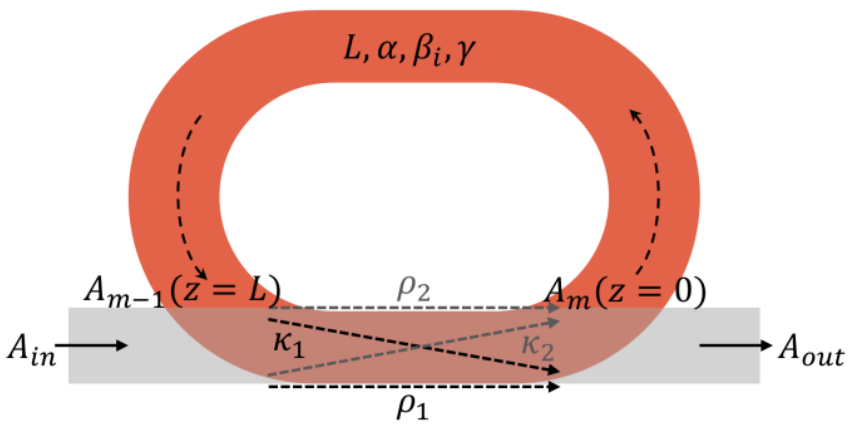

Fig. 1. Schematic of the modelled vertically coupled racetrack resonator: $A_{m}$ represents the intra-cavity field during round-trip $m$ after propagation over the cavity length. $A_{\text {in }}$ and $A_{\text {out }}$ refer respectively to the input and output field at the underlying access waveguide.

Before providing a general description, it is necessary to depict the following assumptions included in our numerical model. We consider an ideal ring resonator that supports a single waveguide mode. It is also assumed that light is linearly polarized and keeps its polarization unchanged at any location of the resonator, which is equivalent to neglecting any impact caused by birefringence during propagation. Hence this model can be applied to any optical resonator in which ignoring these effects is acceptable, regardless of the cavity length or geometry.

The two time-scale approach presented in this paper associates a slow time-scale to the variable $t=m T_{R}$ to the $m$-th round-trip of light circulating inside the resonator with $T_{R}$ the cavity round-trip time, and a fast time-scale to the variable $\tau$ comprised between $-T_{R} / 2$ and $T_{R} / 2$ that describes the temporal distribution of the optical signal circulating inside the cavity.

\section{A. Optical coupler: Transfer function}

Interactions between intra-cavity fields $\left(\tilde{A}_{m} ; \tilde{A}_{m-1}\right)$ and underlying waveguide in the coupling region of the resonator during the passage from round-trip $m-1$ to round-trip $m$ are described by the following relation

$$
\left[\begin{array}{c}
\tilde{A}_{\text {out }} \\
\tilde{A}_{m}(z=0)
\end{array}\right](\omega)=\left[\begin{array}{ll}
\kappa_{1} & \rho_{1} \\
\rho_{2} & \kappa_{2}
\end{array}\right]\left[\begin{array}{c}
\tilde{A}_{m-1}(z=L) \\
\tilde{A}_{\text {in }}
\end{array}\right](\omega)
$$

The matrix representation given above is the so called transfer function of the access coupler applied to the Fourier transform of the slowly varying envelopes of electric fields entering and leaving the coupling region according to Fig. 1. Here, the direct Fourier transform is expressed as: 


$$
\tilde{A}(z, \omega)=\int_{-\infty}^{+\infty} A(z, t) e^{-j \omega t} d t
$$

Complex-valued coefficients $\rho_{i}$ and $\kappa_{i}(i=1$ or 2$)$ correspond respectively to the wavelength-dependent transmission and coupling coefficients of the resonator's access coupler (Fig. 1). Their absolute value determines the amplitude of the transmission and coupling at a given wavelength. The phase of these coefficients accounts for the spectral dependence of the optical path of light in the coupler. In this way, the dispersive properties of the coupling mechanism are properly represented in this numerical framework. In order to respect the conservation of energy, the absolute value of the determinant of the matrix involved in (1) has to be unitary at any wavelength:

$$
\left|\kappa_{1} \kappa_{2}-\rho_{1} \rho_{2}\right|=1
$$

Eq. (3) has been verified a-priori for every case of study presented in this paper.

\section{B. Intra-cavity propagation}

Intra-cavity light propagation can be accurately described by the generalized nonlinear Schrödinger equation for the slowly varying envelope $A(z, \tau)$ of the electric field,

$$
\begin{array}{r}
\frac{\partial A(z, \tau)}{\partial z}=-\frac{\alpha}{2} A(z, \tau)+\left(\sum_{n \geq 2} j^{n+1} \frac{\beta_{n}}{n !} \frac{\partial^{n}}{\partial \tau^{n}} A(z, \tau)\right) \\
+j \gamma\left(1+\frac{j}{\omega_{0}} \frac{\partial}{\partial t}\right)\left[A ( z , \tau ) \left(\left(1-f_{R}\right)|A(z, \tau)|^{2}\right.\right. \\
\left.\left.+f_{R} \int_{-\infty}^{+\infty} h_{R}(s)|A(z, \tau-s)|^{2} d s\right)\right]
\end{array}
$$

where $\tau$ denotes the local time in a so-called moving frame travelling along with the optical envelope at pump frequency group velocity $v_{g}=c / n_{g}$. The relationship between $\tau$ and $t$ is $\tau=t-z / v_{g}$. The phenomena taken into account by GNLSE are: the linear attenuation through coefficient $\alpha$, the dispersion coefficients $\beta_{n}, n \geq 2$ whereas the nonlinear dispersion is taken into account through the simplified optical shock parameter $\tau_{\text {shock }}=1 / \omega_{0}$. Instantaneous Kerr effect manifests itself through the term $\left(1-f_{R}\right)|\mathrm{A}(\mathrm{z}, \tau)|^{2}$. Selfinduced Raman scattering is taken into account by the convolution product between the instantaneous power $|\mathrm{A}(\mathrm{z}, \tau)|^{2}$ and the Raman impulse response.

The GNLSE (4) can be very efficiently solved numerically by a variant of the symmetric split-step method termed the Interaction Picture (IP) method $[27,28]$. Combined with an embedded RungeKutta (RK) scheme with order 4(3) to solve the nonlinear part of the GNLSE, it provides a robust and efficient step-size adaptive numerical method well suited for the purposes of the present study. Details on the RK4(3)-IP method, including a downloadable C++ software can be found in [26].

As the scope of this paper consists in studying the spectral dependence of the coupling effect on KFC formation, we restrict the GNLSE to its NLSE form given by eq. (5) thus limiting propagation effects to linear losses, group-velocity dispersion and Kerr effect.

$$
\begin{gathered}
\frac{\partial A(z, \tau)}{\partial z}=-\frac{\alpha}{2} A(z, \tau)+\sum_{n \geq 2} j^{n+1} \frac{\beta_{n}}{n !} \frac{\partial^{n} A(z, \tau)}{\partial \tau^{n}} \\
+j \gamma A(z, \tau)|A(z, \tau)|^{2}
\end{gathered}
$$

By solving the NLSE (5) along with the boundary conditions imposed by the coupler transfer function (eq. (1)) at each round-trip $m$, the dynamics of a ring resonator can be studied. The system formed by eqs. (1), (5) is referred in the literature as an Ikeda map [24]. For high finesse cavities, these two equations are usually averaged into a driven damped NLS $[15,20]$ which lends itself for analytical developments by neglecting the ability to take into account the frequency dependence of coupling factors and losses in simulations. By keeping the model as described above, it is possible to take into account frequency variations of the coupling and transmission coefficients as well as the nonreciprocity of the cavity and the frequency dependent propagation losses in the context of the simulation of KFC generation and regardless of the cavity quality factor or finesse.

\section{Frequency dependent parameters}

Arlotti et al. have designed in [23] three different access coupling regions intended for $\mathrm{Si}_{3} \mathrm{~N}_{4}$ resonators with $220 \mathrm{GHz}$ FSR to operate in the critical coupling regime over a large frequency bandwidth when pumped in the optical C-band. In reference [23] the parameters that are modified to achieve the desired coupling properties are the access waveguide cross-section dimensions and the separation distance between the $\mathrm{Si}_{3} \mathrm{~N}_{4}$ cavity and the underlying access waveguide. The size, shape and material properties of the cavity remain unchanged. All along this manuscript, in each figure the labels 1 (black curve) and 3 (gray curve) will refer to couplers with phase mismatched structures (PMM) that allow critical coupling over a bandwidth of up to $330 \mathrm{~nm}$ in the best case scenario. The main difference between the two cases labelled 1 and 3 resides in the choice of a different separation distance between ring resonator and access waveguide. For the sake of comparison, label 2 refers to quasi-phase-matched (QPM) structure where the access waveguide and resonator's mode present the same effective index. Unlike the PMM structure, the QPM structure allows for achromatic coupling conditions on a much narrower bandwidth of $23 \mathrm{~nm}$. Observation of $\left|\rho_{i}\right|$ in Fig. 2 clearly indicates the wide and close to critical coupling bandwidth that generally requires $\left|\rho_{i}\right|>0.98$ depending on the wavelength. This bandwidth tends to be one order of magnitude narrower with QPM structure (label 2, red curve). Also, it is worth mentioning that for all three couplers non-zero $\kappa_{i}$ and $\rho_{i}$ arguments are responsible for additive intra-cavity chromatic dispersion. We will come back to this feature and its significant impact on KFC generation in section 2.

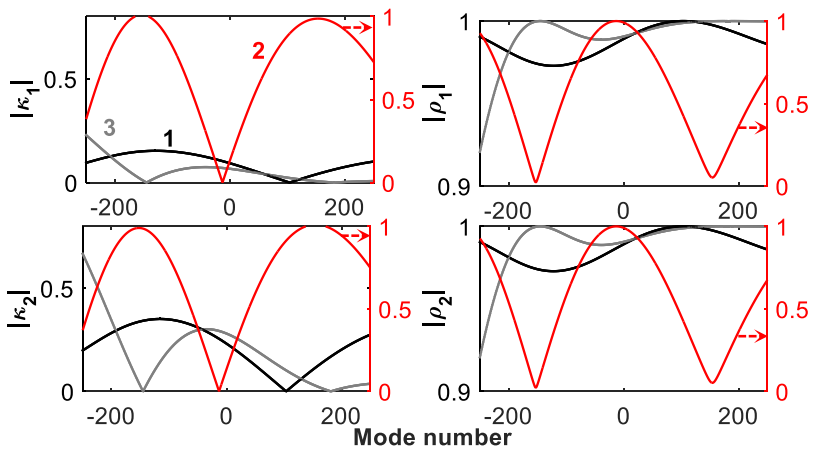

Fig. 2. Absolute values of coupling $\left(\kappa_{1,2}\right)$ and transmission $\left(\rho_{1,2}\right)$ coefficients for each coupler (labelled 1,2 and 3) as a function of simulated optical mode numbers. Labels 1 and 3 (left vertical axis) refer to PMM structure whereas label 2 (right vertical axis) refers to QPM structure (see [23]).

As the material and geometrical properties (e.g. bending radius) of the racetrack resonator have a direct impact on the spectral dependence of dispersion $\left(\beta_{n}\right)$ and propagation losses $(\alpha)$ of the ring resonator [29], we have limited our choice to a single specific design of 
$\mathrm{Si}_{3} \mathrm{~N}_{4} / \mathrm{SiO}_{2}$ racetrack resonator. The spectral dependence of $\alpha$ and $\beta_{2}$ depicted in Fig. 3 illustrates respectively an attenuation coefficient of $29.69\left(\mathrm{~m}^{-1}\right)$ and an anomalous chromatic dispersion of $-18.88\left(\mathrm{ps}^{2} . \mathrm{km}^{-1}\right)$ at $1.55 \mu \mathrm{m}$, the pump laser wavelength. In order to accurately implement these properties into our numerical model, both attenuation and dispersive effects are respectively represented in eq. (5) through a vector expression of $\alpha(\omega)$ and a $10^{\text {th }}$ order Taylor series expansion of $\beta(\omega)$ with coefficients $\beta_{n}$ that matches with the group velocity dispersion (GVD) fluctuations with frequency illustrated in Fig. 3.a. Last, the ring resonator studied in simulations is made up of high confinement $\mathrm{Si}_{3} \mathrm{~N}_{4}$ material characterized by a Kerr coefficient $\gamma=1200\left(W^{-1} \cdot \mathrm{km}^{-1}\right)[13,29]$.

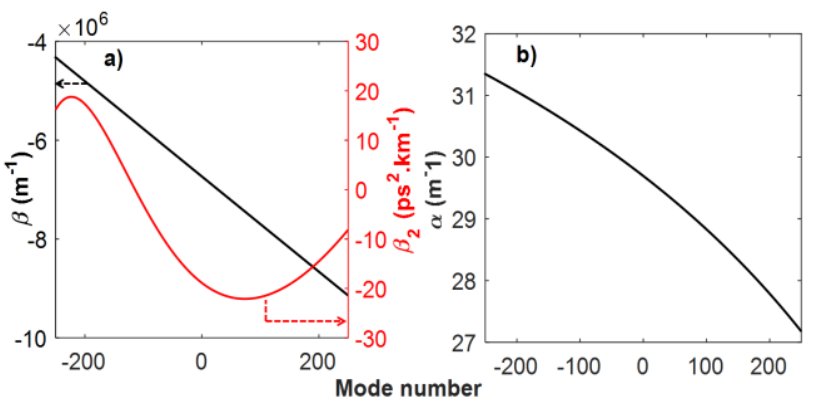

Fig. 3. Representation of the frequency dependent linear coefficients of our modelled $\mathrm{Si}_{3} \mathrm{~N}_{4}$ ring resonator: a) Ring resonator wave vector $\beta$ (black) and group velocity dispersion $\beta_{2}$ (red); b) Material losses are expressed as intensity losses in $\left(\mathrm{m}^{-1}\right)$.

\section{Analytical description of the cold cavity properties}

\section{A. Transfer function}

It is interesting to study the ring resonator behavior in the linear regime i.e. with sufficiently low optical power so that the nonlinear term doesn't manifest itself in the NLSE (see eq. (5)). In this regime, resonator's cavity enhancement properties, resonance linewidth and mode structure can very easily be determined analytically. By assuming no Kerr nonlinearity in (5), intra-cavity field propagation can be described by the following analytical solution,

$$
\tilde{A}_{m}\left(v_{k}\right)=\tilde{A}_{m-1}\left(v_{k}\right) e^{-\left(\frac{\alpha}{2}-j\left(\sum_{n \geq 2} \frac{\beta_{n}}{n !}\left(-2 \pi\left(v_{k}-v_{0}\right)\right)^{n}\right)\right) L}
$$

where $v_{k}$ is the frequency of the mode $k$ in an evenly spaced frequency grid centered around the pumped mode resonance frequency $v_{0}$ so that $v_{k}=v_{0}+k . F S R(k \in \mathbb{Z})$. By combining relation (6) with the coupler's transfer function (1), the resonator's transfer function $T(\varphi)$ is easily determined in the steady state regime at any given wavelength and is expressed as,

$$
T(\varphi)=\frac{\tilde{A}_{\text {out }}}{\tilde{A}_{\text {in }}}=\frac{\rho_{1}+\left(\kappa_{1} \kappa_{2}-\rho_{1} \rho_{2}\right) \widehat{L} \widehat{D} e^{j \varphi}}{1-\rho_{2} \widehat{L} \widehat{D} e^{j \varphi}}
$$

where ring propagation losses and dispersion are respectively modelled by operators $\widehat{L}=e^{-\frac{\alpha}{2} L}$ and $\widehat{D}=e^{+j\left(\sum_{n \geq 2} \frac{\beta n}{n !}\left(-2 \pi\left(v_{k}-v_{0}\right)\right)^{\mathrm{n}}\right) \mathrm{L}}$. The variable $\varphi$ represents the linear phase accumulated by the intracavity field over one round-trip with respect to the FSR spaced frequency grid. This linear phase is linked to the shift in frequency $f \in[-F S R / 2 ; F S R / 2]$ which represents the frequency detuning in the vicinity of $\mathrm{k}^{\text {th }}$ optical mode: $k 2 \pi+\varphi=2 \pi \frac{v_{k}+f}{F S R}$.

\section{B. Quality factor and power enhancement factor}

Maximizing the resonator's quality (Q) factor over the widest achievable bandwidth favors the KFC generation process by reducing the total round-trip losses that have to be overcome by parametric gain in order for modulation instability to manifest itself and give rise to an optical frequency comb. The resonator's $Q$ factor is a measure of round-trip losses and is linked to the full-width at half maximum $\Delta f_{1 / 2}$ of the squared absolute value of the cavity's transfer function $|T(\varphi)|^{2}$ at frequency mode $v_{k}$ and thus, gives useful information pertaining to the selectivity of the cavity. The quality factor at a given frequency $v_{k}$ is expressed as follows:

$$
Q=\frac{v_{k}}{\Delta f_{1 / 2}}
$$

In this numerical work, by using relation (7), it is possible to compute the local $\mathrm{Q}$ factor at each resonance all along the simulated frequency span and for each coupling scheme. As depicted in Fig. 4, whatever the coupling design is, a similar $Q$ factor $\approx 1.410^{5}$ is observed at pumping wavelength. However, one can appreciate how the coupling design drastically affects Q-factor value with frequency. It is easy to distinguish the case corresponding to QPM structure (coupler 2) as it corresponds to a high quality factor over a considerably narrower bandwidth compared to that of the two other cases.

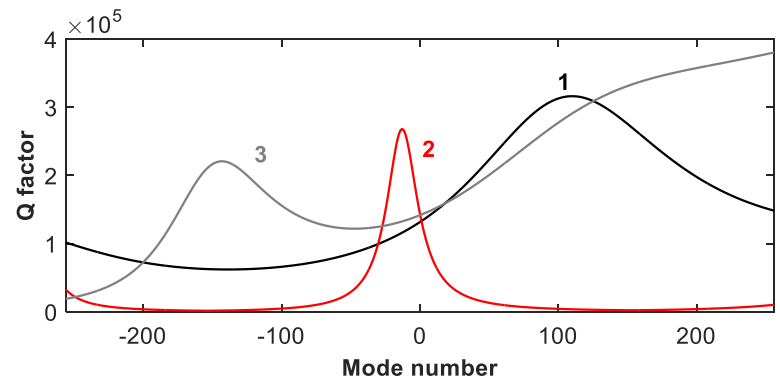

Fig. 4. Frequency-dependence of $Q$ factor for each of the different access coupling regions described in section 1 . Labels 1 and 3 refer to PMM coupling structure whereas label 2 refers to QPM structure.

To favor KFC generation in optical resonators it is also important to optimize the pump laser energy storage in the ring cavity. Pump energy storage efficiency is quantified through the ring cavity power enhancement factor (PEF) defined as the ratio intra-cavity power at resonance over input pump power. An analytical expression of a resonator's PEF as a function of its coupling and intra-cavity properties can be deduced from (1) and (6) and it is given by,

$$
P E F=\frac{\left|\tilde{A}_{m}\right|^{2}}{\left|\tilde{A}_{\text {in }}\right|^{2}}=\frac{\left|\kappa_{2}\right|^{2}}{\left|1-\hat{L} \rho_{2}\right|^{2}}
$$

The PEF is a function of $\kappa_{2}$ and $\rho_{2}$ coefficients, as a consequence it is a function of frequency. PEF achieved with each coupler design is depicted below in Fig. 5.

As illustrated in Fig. 5, PEF strongly differs for each case of study. A maximum PEF of 210 at pump wavelength is obtained with the third coupler design. The simultaneous observation of $\mathrm{Q}$ factor and PEF as function of frequency confirm that PMM coupler structure and more precisely the third design will favor KFC generation as it guarantees a maximum $\mathrm{PEF}$ at the desired pump wavelength and optimum $\mathrm{Q}$ factor over the largest bandwidth that minimizes degenerate FWM power threshold, by means of minimizing total round-trip losses. For passive resonators, it is of greatest interest to design the coupler so that 
resonator's critical coupling is obtained in the vicinity of pump wavelength, while maintaining a high quality factor elsewhere.

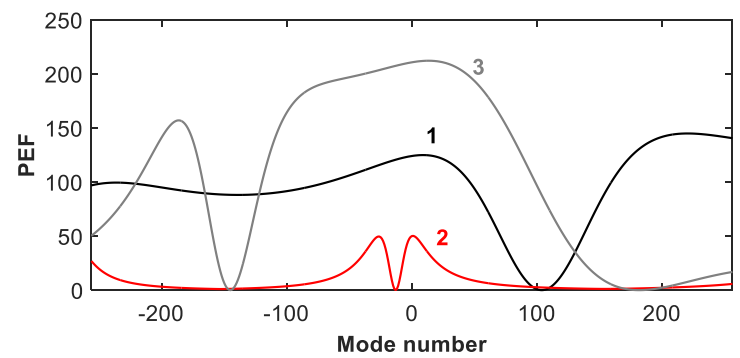

Fig. 5. Resonator's PEF as a function of frequency for each access coupler design described in section 1.C.

\section{Cold cavity resonance mismatch}

Chromatic dispersion affects the spacing between resonator's modes and thus creates resonance mismatch. In optical resonators, dispersion can result from whispering gallery modes, meaning that some modes propagate at the outer edges of the resonator while other propagate closer to the inner edge of the racetrack waveguide (or closer to the center of the cavity in the case of mini-disks, microspheres...) and material dispersion which is the frequency dependence of a material's refractive index. Dispersion plays a crucial role in KFC generation because it allows phase matching to occur between the comb spectral components thanks to Kerr effect.

Intra-cavity and coupler dispersion are taken into account separately in the model presented in this paper. Intra-cavity dispersion accounts for all sources of dispersion during propagation in the ring cavity. It is modeled by the $\beta_{n}$ coefficients in the propagation eq. (5). Hence, the cold cavity resonance frequency mismatch $\Delta v_{c}$ induced by intra-cavity propagation can be deduced from the dispersion coefficients $\beta_{n}$ by the relation

$$
\Delta v_{c}=\frac{F S R}{2 \pi}\left(\sum_{n \geq 2} \frac{\beta_{n}}{n !}\left(-2 \pi\left(v_{k}-v_{0}\right)\right)^{n} L\right)
$$

However in eq. (10) $\Delta v_{c}$ does not describe the effects of dispersion imposed to the intra-cavity signal when it crosses the access coupler.

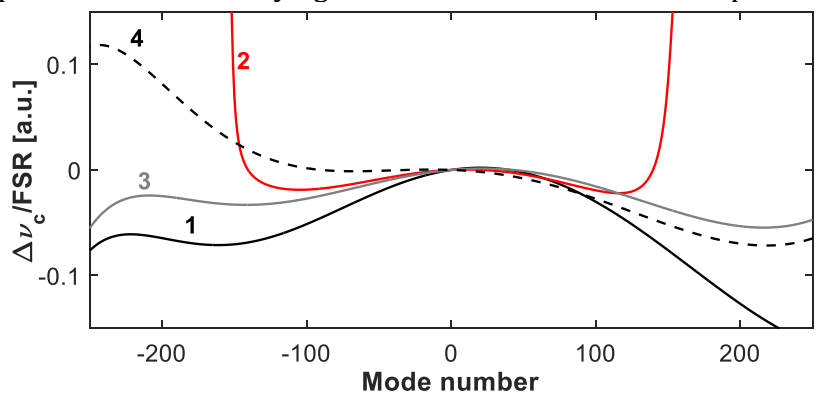

Fig. 6. Cold resonance frequency mismatch for the three resonators under study, taking into account all sources of dispersion (coupler and intra-cavity dispersion). The dashed line labelled 4 represents the resonance frequency mismatch caused by dispersion during intracavity propagation only for comparison. Dispersion coefficients are taken into account up to the tenth order.

To that effect, eq. (7) can be used to determine the exact position of the resonance peaks associated to all the simulated resonator modes, thus giving a resonator's cold resonance frequency mismatch taking into account total round-trip dispersion consisting of both, intra-cavity and coupler dispersion.
The cold resonance frequency mismatch of each resonator normalized with respect to the cavity FSR is represented in the above Fig. 6. The effects of intra-cavity dispersion alone are also represented with a dashed curve 4 for comparison purposes. The importance of taking coupler dispersion into account in our simulations becomes apparent as the mode structure of each resonator is strongly shaped by its access coupler design, further highlighting the importance of the work performed in reference [23].

In [30] Bao et al. studied the effect of mode pulling due to the Kerr effect in nonlinear resonators and they demonstrated that, in the formation of cavity solitons inside an optical resonator, Kerr-induced mode-pulling is responsible for pulling the cold cavity resonance into an evenly-spaced resonance mode structure that produces appropriate and stable phase matching conditions for the existence of a KFC in the resonator. In fact, the demonstration provided by Bao et al. can be reproduced with the model presented in this paper by applying it to eq. (5) instead of the Lugiato-Lefever equation used in [30]. By taking the Fourier transforms of the intra-cavity propagation equation (5), one obtains,

$$
\begin{aligned}
& \frac{\partial \tilde{A}(z, \omega)}{\partial z}=-\frac{\alpha}{2} \tilde{A}(z, \omega) \\
& +j \sum_{n \geq 2} \frac{\beta_{n}}{n !}\left(-\left(\omega-\omega_{0}\right)\right)^{n} \tilde{A}(z, \omega) \\
& +j \gamma \operatorname{Re}\left\{\tilde{A}(z, \cdot) \otimes \tilde{A}(z, \cdot) \otimes \tilde{A}^{*}(z, \cdot)\right\}(\omega) \\
& -\gamma \operatorname{Im}\left\{\tilde{A}(z, \cdot) \otimes \tilde{A}(z, \cdot) \otimes \tilde{A}^{*}(z, \cdot)\right\}(\omega)
\end{aligned}
$$

where $\tilde{A}^{*}$ denotes the complex conjugate of $\widetilde{\mathrm{A}}$, the Fourier transform of the complex envelope $A$, and $\otimes$ denotes the convolution product. In eq. (11) one can identify the phase modulation terms due to intra-cavity dispersion $j \sum_{n \geq 2} \frac{\beta_{n}}{n !}\left(-\left(\omega-\omega_{0}\right)\right)^{n}$ (Fig. 6 dashed line) and more importantly the phase modulation term due to nonlinear effect $j \gamma \operatorname{Re}\left\{\widetilde{\mathrm{A}}(\mathrm{z}, \cdot) \otimes \widetilde{\mathrm{A}}(\mathrm{z}, \cdot) \otimes \tilde{A}^{*}(\mathrm{z}, \cdot)\right\}(\omega) / \widetilde{\mathrm{A}}(\omega, \tau)$ that is responsible for the compensation of all phase mismatch effects when a KFC is generated inside the cavity. This nonlinear term is linked to the Kerrinduced resonance mismatch in frequency units

$$
\Delta v_{k}=\frac{F S R}{2 \pi} \frac{\gamma \operatorname{Re}\left\{\tilde{A}(z, \cdot) \otimes \tilde{A}(z, \cdot) \otimes \tilde{A}^{*}(z, \cdot)\right\}(\omega)}{\tilde{A}(\omega, \tau)}
$$

To conclude, the total residual hot resonance mismatch $\left(\Delta v_{h}\right)$ of a resonator when power is circulating inside the optical cavity is the sum of the cold resonance mismatch $\left(\Delta v_{c}\right)$ and Kerr-induced resonance mismatch $\left(\Delta v_{k}\right)$ :

$$
\Delta v_{h}=\Delta v_{c}+\Delta v_{k}
$$

Hence, $\Delta v_{h}$, quantifies how much each resonator mode is pulled into an equally spaced frequency grid. For a given mode, a small residual mismatch means that the cavity resonance is aligned with the corresponding comb component being generated close to that mode. Since coupler dispersion is actually a supplementary source of dispersion in our study (Fig. 6 curves 1, 2 and 3), the nonlinear phase modulation term has to compensate the total resonator dispersion and not only the dispersion phase modulation term given in eq. (11).as: $j \sum_{n \geq 2} \frac{\beta_{n}}{n !}\left(-\left(\omega-\omega_{0}\right)\right)^{n}$.

When a wide and stable frequency comb is generated in an optical resonator, Kerr induced resonance mismatch compensates the cold cavity resonance mismatch producing a hot cavity mode structure that is aligned with the generated comb teeth. As $\Delta v_{k}$ is directly determined by the Fourier transformed intra-cavity field (12), and $\Delta v_{h}$ has to be sufficiently small in order for the generated modes to oscillate freely in the resonator, $\Delta v_{c}$, and thus dispersion, determines the comb 
shape that can be expected to be generated in a given resonator as it determines the necessary phase matching conditions to be satisfied.

From the resonance mismatch curves presented in Fig. 6, we can determine that resonator 2 has the best suited dispersive properties for the generation of KFCs since it has the flattest mismatch curve out of the three resonators and the only one that presents zero-crossing points (around modes -140 and +140 ) suggesting the possibility of dispersive wave emission around these spectral regions [31]. With a frequency-independent quality factor, it is the dispersive properties of a resonator that determines the shape and the bandwidth of a frequency comb for a given pump power [31]. This same resonator, however, presents a narrow-band high quality factor (Fig. 4) around the pumped mode that strongly degrades as the mode number increases, reaching minima of the order of 1500 around mode -140 and 2200 around mode 140, all of which will hinder the comb generation and spreading process as will be seen in the section to come. Resonators 1 and 3 have similar cold mismatch structures but resonator 3 has a flatter one which thus favors wider frequency combs than resonator 1.

\section{Simulation results and discussion}

$\mathrm{KFC}$ generation simulations rely on the iterative model presented in section 1, where equations (1) and (5) serve as the basis for numerical simulations. Pump laser detuning with respect to the pumped resonator mode is a crucial parameter in Kerr comb generation. Indeed, due to the Kerr effect, the refractive index of a nonlinear optical cavity increases as optical power is accumulated, lengthening the optical path of light which results in a shift of the cavity resonance towards lower frequencies. The frequency detuning of the pumped laser has to be controlled in such a manner that it follows the Kerr induced pumped resonance shift to efficiently store the necessary power levels in the resonator that lead to Kerr frequency comb excitation [3].

To that effect, a cw pump laser of average power $P_{0}$ as described in the temporal domain by,

$$
A_{\text {in }}(\tau)=\sqrt{P_{0}} e^{j \delta}+n(\tau)
$$

is used in simulations. The frequency of the pump laser is initially blue-detuned with respect to the cold cavity resonance and linearly shifted towards lower frequencies across the cold cavity resonance via the phase detuning parameter $\delta$.

An additive white Gaussian noise source $n(\tau)$, that models laser spontaneous emission, $150 \mathrm{~dB}$ weaker than the cw pump laser is used as a seeding signal for degenerate four wave mixing (FWM) interactions that take place in the initial stages of KFC generation [32].

For the sake of comparison, $P_{0}$ has been adapted for each resonator properties described in section 2 so that the stored intra-cavity pump power represented by the product $\operatorname{PEF}\left(v_{0}\right) P_{0}$ remains constant despite their different $P E F\left(v_{0}\right)$. Hence, because of this normalization, the differences observed in KFC formation may only be attributed to discrepancies in frequency-dependent Q-factor and dispersive properties of each resonator. The pump power has been adjusted to $1.7,4.2$ and $1 \mathrm{~W}$ for resonators 1,2 and 3 respectively.

Fig. 7.a represents intra-cavity power accumulation in the absence of nonlinear effects as the pump laser frequency is swept through the pumped mode resonance. The Lorentzian shape of the resonances is maintained and maximum power accumulation is the same for each resonator. The minor variation in resonance half-width is a result of the slight difference in quality factor at pumped mode of each resonator (cf. Fig.4). Fig. 7.b shows power accumulation in each resonator when the Kerr effect is taken into account but in the absolute absence of noise (i.e. seeding signal $n(t, \tau)=0$ ). In the absence of perturbations, no degenerate FWM interactions (MI) can take place in the cavity. Due to the pump power normalization used in simulations, power accumulation remains the same for each cavity because selfphase modulation is the only nonlinear effect taking place in this specific instance. As a conclusion, by normalizing pump power, i.e. by keeping the product $P E F\left(v_{0}\right) P_{0}$ constant, any differences that will be observed in $\mathrm{KFC}$ generation dynamics and its steady-state profile will be attributed to factors other than the PEF at the pumped mode.

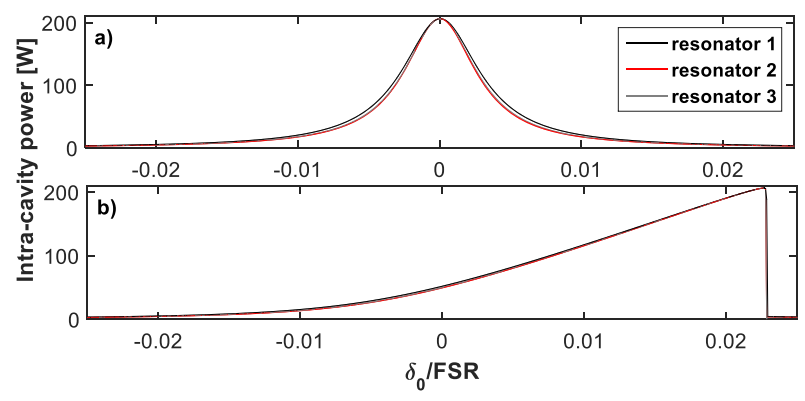

Fig. 7. Average intra-cavity power storage for each resonator design as pump laser wavelength is scanned over the cold cavity resonance from shorter to longer wavelengths. a) case of a dispersive cavity only $\left.\beta_{n} \neq 0, \gamma=0 ; \mathrm{b}\right) \beta_{n} \neq 0, \gamma \neq 0, n(t, \tau)=0$.

Fig. 8.a shows the intra-cavity average power accumulation during laser scan simulations for each resonator with normalized pump power, including the nonlinear Kerr effect and using a seeding signal that allows for modulation instability (MI) and FWM interactions to take place. Intra-cavity power storage dynamics are shown to be highly dependent on the quality-factor profile and the dispersive properties of each nonlinear resonator. Unlike resonator 2 , the intra-cavity power accumulation dynamics of resonators 1 and 3 strongly resembles the conventional profile observed when parametric amplification occurs. Indeed, one can correlate the identified marks in Fig. 8.a to well-known functioning regimes shown in Fig. 8.b that subsequently appear during pump laser frequency scan.

In Fig. 8.b, inset I refers to the temporal and spectral profile corresponding to the apparition of the first modulation sidebands in each resonator. Further increasing the detuning leads to the formation of temporal patterns shown in inset II [33]. These patterns correspond to a mode-locked frequency comb with a spacing corresponding to an integer number of cavity FSRs. With much larger detuning, more power is accumulated in the cavity thus leading to the broadening of the sidebands generated in II until they overlap and fill the resonator modes between them [32]. This conducts to the formation of a broad but incoherent FSR-spaced frequency comb labelled as the chaotic MI regime (Fig. 8.b, inset III). Inset IV depicts the stable multiple pulses regime where temporal pulses with constant space between them circulate in the cavity. The generated spectrum is not smooth but stable. It results from the superposition of the respective comb spectrum associated to each pulse circulating at the same repetition rate.

In the case of resonator 2, the average intra-cavity power accumulation dynamic (Fig. 8.a.2) does not resemble those observed for its counterparts (resonators 1 and 3). Indeed, the average power accumulation profile shows a sharp drop around the 0.01 normalized detuning mark. The resonance shift induced by self-phase modulation is observed in resonator 2 . However, due to strong losses, parametric nonlinear effects are strongly degraded and power exchanges between the pumped mode and the generated comb are weak. This leads to a power accumulation trace that more closely fits the one observed in Fig. 7.b. 

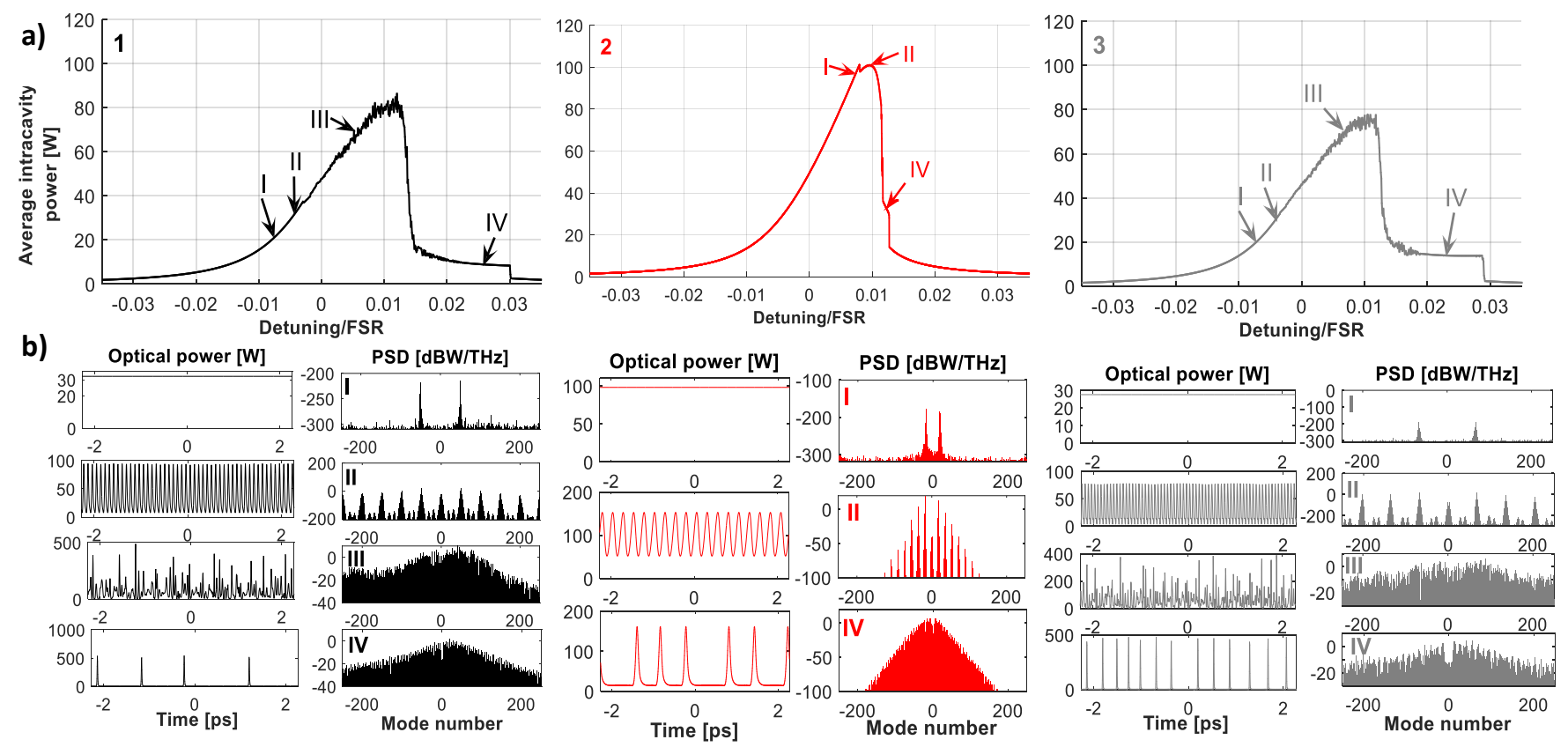

Fig. 8. Numerical results showing the Kerr comb generation process in resonators 1, 2 and 3. a) Average intra-cavity power as a function of frequency detuning during laser scan simulations. b) Intra-cavity field time-domain power distribution and power spectrum density (PSD) at different stages during laser scan simulations: I- Apparition of the first modulation sidebands. II- Formation of temporal patterns. III- Chaotic MI regime. IV- Stable multiple pulses regime.

We can see from inset I that the appearance of the first modulation sidebands occurs only when a cw optical power of around $95 \mathrm{~W}$ is circulating inside the optical cavity, as opposed to resonators 1 and 3 where less than $30 \mathrm{~W}$ is enough to excite modulation instability. In addition to this, these MI generated sidebands are formed close to the pumped mode, around modes \pm 18 as opposed to resonators 1 and 3 where the first MI sidebands are generated around modes \pm 50 and \pm 68 respectively. Indeed, as depicted in Fig. 4, resonators 1 and 3 possess quality factor profiles that maintain the same order of magnitude over a wide spectral region. On the other hand, resonator 2 is characterized by a narrowband high quality factor around the pumped mode that suffers strong degradations with frequency and a contrast of a factor of 97 between the quality factor at the pumped mode and the lowest quality factor in the simulated spectral domain. As the parametric gain engendered by the pump has to overcome the resonator's losses in order for MI to occur, a substantially higher power (more than 3 times the necessary circulating power in resonators 1 and 3 ) is needed in resonator 2 to initiate the comb generation process because of the fast degradation of total quality factor (round-trip losses) as the frequency increases. Furthermore, after the formation of temporal patterns in resonator 2 , the chaotic MI regime does not seem to take place with the pump power used in simulations. The comb regime in resonator 2 immediately goes from temporal patterns (inset II, resonator 2) to a stable train of pulses (inset IV, resonator 2) after the drop in average intra-cavity power around the 0.01 normalized detuning mark mentioned above.

Although frequency scan simulations may potentially be comparable with experiments, they do not always yield the single soliton regime which may require numerous laser scan simulations in order to be generated and in some cases may not be accessible at all in this manner. Alternatively, different approaches exist in order to reliably achieve the single soliton regime. From an experimental standpoint, another method such as the pump laser forward then backward tuning has been shown to be effective in controlling the number of pulses generated in the cavity [34]. From a theoretical standpoint, the use of a single-shot pulse to deterministically trigger the single soliton state in an optical resonator has recently been studied and could present an interesting alternative to frequency scan simulations conventionally used in practice [35].

The hard excitation method consisting in performing sudden changes in pump power or frequency detuning; as was initially proposed by Matsko et al. in [36] is the method we used in this study in order to obtain single pulse solutions supported by each resonator and depicted in Fig. 9. For each resonator, regular frequency scan simulations are performed until the chaotic MI regime is achieved. Then, an abrupt detuning of the pump laser frequency is performed towards lower frequencies. By appropriately choosing the frequency detuning offset, the single pulse regime and its associated smooth spectrum are obtained. This allows for better interpretations than the multiple pulses spectra shown in Fig. 8.b (inset IV).

Fig. 9 shows the single soliton spectra generated in this fashion along with the cold resonance mismatch for resonators 1 and 3 and the frequency-dependent $\mathrm{Q}$ factor for resonator 2 . It becomes clear in Fig. 9.a and Fig. 9.c that the spectrum associated with the generated pulse is determined by the dispersive properties of the resonator (cold resonance mismatch) when $\mathrm{Q}$ factor fluctuations are not significant as evidenced by resonators 1 and 3. However, Fig. 9.b shows that a strong $\mathrm{Q}$ factor degradation can, in fact, degrade the spectral occupation of the generated KFC by acting as losses for the generated comb lines. Indeed, photons generated through parametric nonlinear effects are less efficiently stored in spectral regions where $Q$ factor is low. In the case of resonator 2, excessive round-trip losses are due to extrinsic $Q$ factor degradation through the sharp fluctuation of output coupling coefficient $\kappa_{1}$ (see Fig. 2).

Here, the sharp contrast of $Q$ factor seems to limit the bandwidth of the generated spectrum and further modifies comb shape in addition to the dispersion properties of the cavity and access coupler. The dispersive properties of resonator 2 (cf. Fig.6) even suggest the 
potential for the generation of dispersive waves around modes -140 and 140 that would further increase comb bandwidth. However, with losses ( $Q$ factor) being strongest (lowest) around these spectral modes, this effect does not take place.

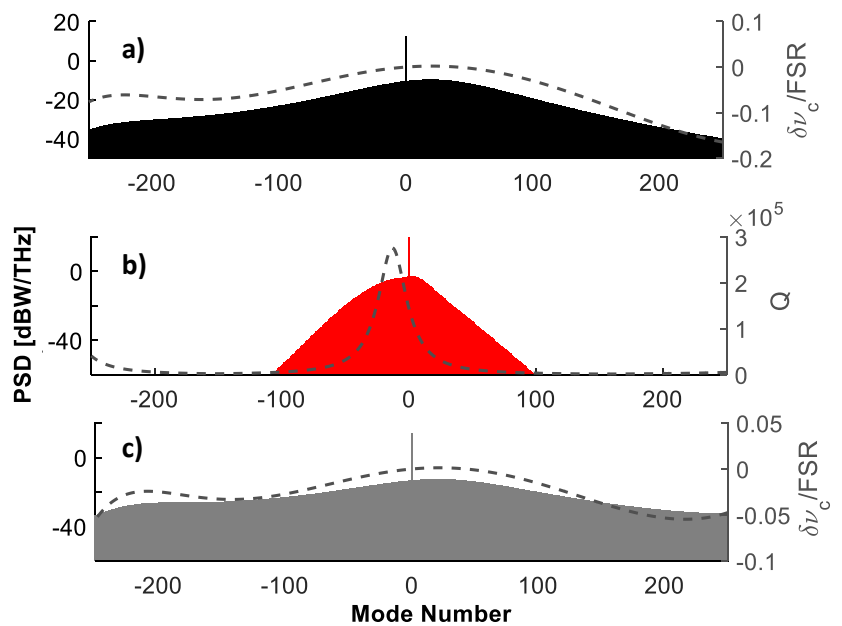

Fig. 9. Intra-cavity single pulse PSD supported by each simulated resonator. The power spectral density associated to each pulse is represented with respect to the left vertical axis. Normalized cold resonance mismatch for resonators 1 (a) and 3 (c) and Q factor for resonator 2 (b) are represented by the dashed line along the right vertical axis.

It is however important to recall that the bandwidth and shape of the generated KFC will be mainly dictated by the dispersive properties of the resonator as a whole because the generated comb has to "fit" in the resonator's mode structure as evidenced by equations (11), (12) and (13) by producing an effective hot resonance mismatch $\Delta v_{h}$ that is small enough so that parametric interactions between comb lines can take place. Degradations with frequency of total resonator losses $(Q$ factor), can induce additional changes to comb bandwidth and shape when significant enough, as evidenced mainly by case 2 studied in this paper. In parallel the comb initiation process can be hindered as evidenced by the frequency scan trace of resonator 2 in Fig. 8.a.2. Similarly, we could expect that an increase in $Q$ factor around the parametric sidebands engendered by the pump laser could decrease the power threshold for comb generation by means of reducing roundtrip losses.

Fig. 10 illustrates the hot resonance residual mismatch $\Delta v_{h}$ during laser scan simulations for resonator 3 . This phase-mismatch curve is obtained using eq. (13) at different stages during laser scan simulations. During the apparition of the first modulation sidebands and temporal patterns, the flat regions observed in insets I and II coincide perfectly with the spectral regions where sidebands are being generated suggesting stable FWM interactions between spectral components (see Fig. 8.b insets I and II for resonator 3). However, no stable phase matching takes place during the chaotic regime as it was previously reported in [30]. In this regime, power exchanges between the spectral components of the comb take place in an apparently random (chaotic) fashion. Hence the chaotic distribution of the temporal intra-cavity field and phase of its spectral components. Inset IV shows the phase matched mode structure of the hot resonance in the stable pulsed regime. The residual hot cavity resonance mismatch observed in this figure remains flat over a large bandwidth and shows that phase matching (cavity mode alignment) is achieved over a large bandwidth

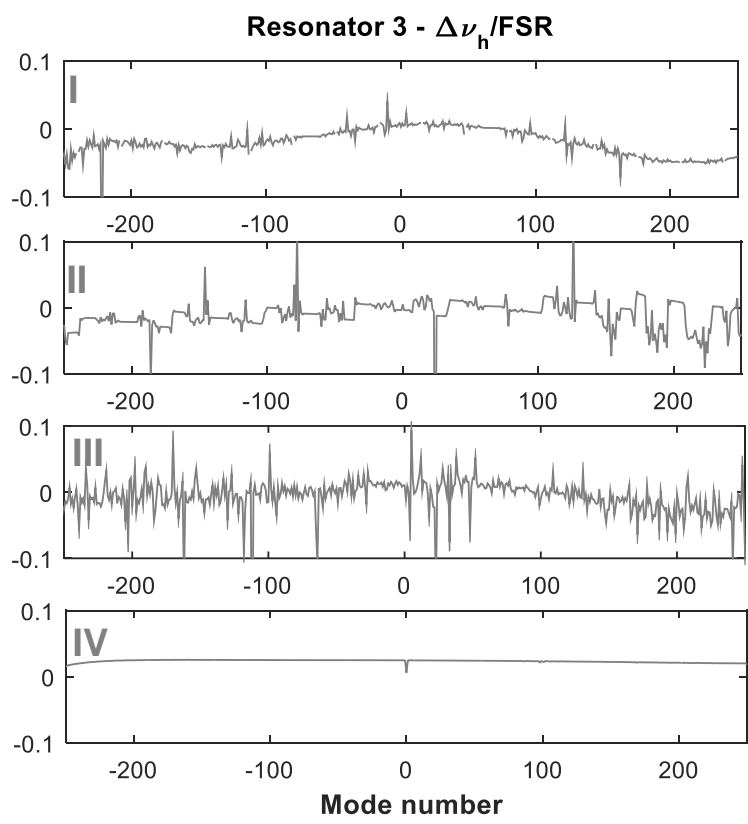

Fig. 10. Evolution of the hot resonance mismatch $\Delta v_{h}$ during laser scan simulations for resonator 3 . Insets I through IV correspond to the stages of comb generation presented in Fig. 8.

Last but not least, an important aspect to consider when working with vertically coupled structures is the shaping of the output signal induced by the resonator's coupler. The fact that $\kappa_{1}$ is frequencydependent will inherently modify the outgoing spectrum shape. This particular effect is of significant importance when the output spectrum no longer replicates its intra-cavity counterpart as evidenced by Fig. 11 in comparison with Fig. 9. This issue is of particular importance in the context of coupler design since it most certainly will modify the shape of the only accessible signal in practice.

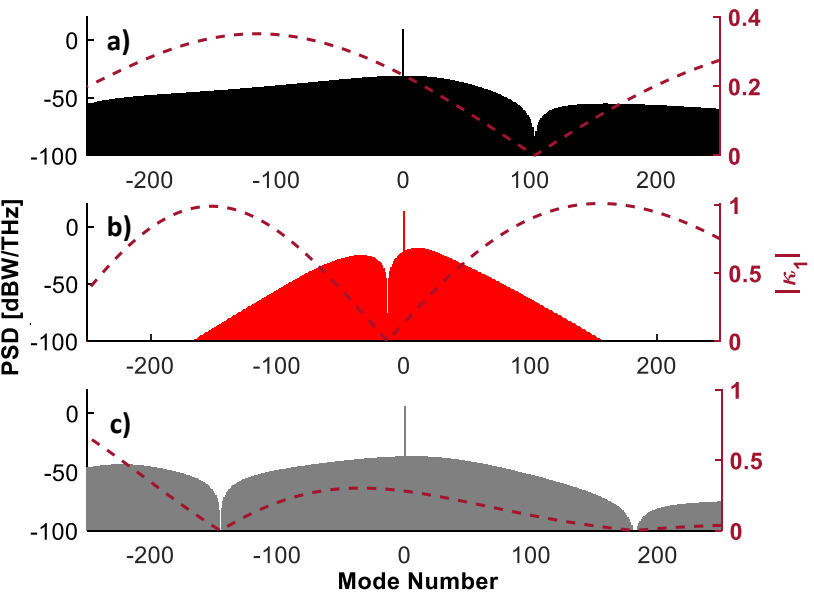

Fig. 11. Output single pulse PSD (left vertical axis) superimposed with the output coupling coefficient $\kappa_{1}$ of each respective resonator's access coupler : 1 (a), 2 (b) and 3 (c)

\section{Conclusion}

We have developed a numerical tool based on the Ikeda map for KFC generation in vertically coupled $\mathrm{Si}_{3} \mathrm{~N}_{4}$ microresonators that eases the implementation of spectral dependence in the coupling and dispersive properties of the coupling design. 
Based on a selection of three different coupling schemes dedicated to produce a wide-band critically coupled resonator we have numerically highlighted and analytically interpreted strong discrepancies in the KFC generation process and steady-state profile showing a strong impact of quality factor as well as resonator's mode structure. Indeed, modifications of PEF at the pumped resonator mode impact comb generation power threshold through pump power accumulation efficiency. However, even for normalized pump power accumulation dynamics, simulations show that the comb generation process is highly dependent on the spectral profile of the resonator's Q factor when a strong contrast occurs between the pumped mode and parametric gain regions (resonator 2). When $\mathrm{Q}$ factor remains of the same order of magnitude over the simulated bandwidth (resonators 1 and 3), comb generation dynamics and shape are mainly dictated by the resonator's mode structure i.e. its dispersive properties. Further flattening the cavity mode structure would lead to the generation of a wider spectra [14,37]. Hence careful attention has to be paid in the design of access coupler as it can result in degradation of $Q$ factor and mode structure with frequency especially with the intent of generating KFC.

Our numerical model could serve as a benchmarking tool with alternative $\mathrm{Si}_{3} \mathrm{~N}_{4}$ ring resonators based on horizontal coupling schemes which are nowadays widely deployed [15]. Besides, our technique could apply to alternative technologies like silica micro-sphere or $\mathrm{MgF}_{2}$ mini-disk since a spectral description of the coupler is achievable. However, the simplifying approximations described in section 1 need to be acceptable and nonlinearity should be limited to Kerr effect. Indeed, according to specific conditions, stimulated Brillouin scattering (SBS) may occur if the ring resonator's Brillouin frequency shift is a multiple of FSR [38], and severely affect the KFC generation dynamic. Implementation of additive SBS in the ring resonator would need heavy modification of the ring propagation equation into respective coand contra- propagating equation and acoustic phonon rate equation [39]. Also, Brillouin backscattering effect may happen when tapered fiber is employed as access coupling waveguide because of strong mode effective area reduction [40]. Other undesirable nonlinear effect like stimulated Raman scattering (SRS) may occur and disturb the Kerr comb generation too [41]. Contrary to SBS the SRS effect could be rapidly numerically computed by replacing propagation equation (5) with the GNLSE (4) [26, 27].

In addition to this, the implementation of a simulation method that separates intra-cavity propagation from access coupling limits the intra-cavity propagation step-size to a maximum value that equals the cavity round-trip length. Within the scope of the experimental cases presented in this paper i.e. for resonators showing a $\mathrm{Q}$ factor ranging from $10^{5}$ to $10^{6}$, this limitation doesn't bring a time consuming computation effort. However for higher $\mathrm{Q}$ factors i.e. for resonators showing a longer storage time, one could expect simulation time to become significantly higher. A different approach such as the coupled mode equations approach may suit better [22]. Nonetheless, we expect our numerical investigations to be useful and bring a better understanding in Kerr comb generation.

\section{References}

1. T. W. Hänsch, "Nobel Lecture: Passion for precision," Rev. Mod. Phys. 78, 1297-1309 (2006).

2. J. Pfeifle, V. Brasch, M. Lauermann, Y. Yu, D. Wegner, T. Herr, K. Hartinger, P. Schindler, J. Li, D. Hillerkuss, R. Schmogrow, C. Weimann, R. Holzwarth, W. Freude, J. Leuthold, T. J. Kippenberg, and C. Koos, "Coherent terabit communications with microresonator Kerr frequency combs," Nat. Photonics 8, 375-380 (2014).
3. T. Herr, V. Brasch, J. D. Jost, C. Y. Wang, N. M. Kondratiev, M. L. Gorodetsky, and T. J. Kippenberg, "Temporal solitons in optical microresonators," Nat. Photonics 8, 145-152 (2014).

4. Z. Jiang, C.-B. Huang, D. E. Leaird, and A. M. Weiner, "Optical arbitrary waveform processing of more than 100 spectral comb lines," Nat. Photonics 1, 463-467 (2007).

5. M. T. Murphy, T. Udem, R. Holzwarth, A. Sizmann, L. Pasquini, C. Araujo-Hauck, H. Dekker, S. D’Odorico, M. Fischer, T. W. Hänsch, and others, "High-precision wavelength calibration of astronomical spectrographs with laser frequency combs," Mon. Not. R. Astron. Soc. 380, 839-847 (2007).

6. J. Morgenweg, I. Barmes, and K. S. E. Eikema, "Ramsey-comb spectroscopy with intense ultrashort laser pulses," Nat. Phys. 10, 30-33 (2014).

7. W. Liang, D. Eliyahu, V. S. Ilchenko, A. A. Savchenkov, A. B. Matsko, D. Seidel, and L. Maleki, "High spectral purity Kerr frequency comb radio frequency photonic oscillator," Nat. Commun. 6, 7957 (2015).

8. M. C. Stumpf, S. Pekarek, A. E. H. Oehler, T. Südmeyer, J. M. Dudley, and U. Keller, "Self-referencable frequency comb from a 170-fs, 1.5- $\mu \mathrm{m}$ solid-state laser oscillator," Appl. Phys. B 99, 401408 (2010).

9. V. Huet, A. Rasoloniaina, P. Guillemé, P. Rochard, P. Féron, M. Mortier, A. Levenson, K. Bencheikh, A. Yacomotti, and Y. Dumeige, "Millisecond Photon Lifetime in a Slow-Light Microcavity," Phys. Rev. Lett. 116, (2016).

10. I. S. Grudinin, N. Yu, and L. Maleki, "Generation of optical frequency combs with a CaF 2 resonator," Opt. Lett. 34, 878-880 (2009).

11. R. Henriet, G. Lin, A. Coillet, M. Jacquot, L. Furfaro, L. Larger, and Y. K. Chembo, "Kerr optical frequency comb generation in strontium fluoride whispering-gallery mode resonators with billion quality factor," Opt. Lett. 40, 1567 (2015).

12. P. S. Donvalkar, A. Savchenkov, and A. Matsko, "Self-injection locked blue laser," J. Opt. 20, 045801 (2018).

13. J. S. Levy, A. Gondarenko, M. A. Foster, A. C. Turner-Foster, A. L. Gaeta, and M. Lipson, "CMOS-compatible multiple-wavelength oscillator for on-chip optical interconnects," Nat. Photonics 4, 3740 (2010).

14. S. Kim, K. Han, C. Wang, J. A. Jaramillo-Villegas, X. Xue, C. Bao, Y. Xuan, D. E. Leaird, A. M. Weiner, and M. Qi, "Dispersion engineering and frequency comb generation in thin silicon nitride concentric microresonators," Nat. Commun. 8, (2017).

15. Y. Okawachi, K. Saha, J. S. Levy, Y. H. Wen, M. Lipson, and A. L. Gaeta, "Octave-spanning frequency comb generation in a silicon nitride chip," Opt. Lett. 36, 3398 (2011).

16. S. Coen, H. G. Randle, T. Sylvestre, and M. Erkintalo, "Modeling of octave-spanning Kerr frequency combs using a generalized meanfield Lugiato-Lefever model," Opt. Lett. 38, 37 (2013).

17. Y. K. Chembo and C. R. Menyuk, "Spatiotemporal Lugiato-Lefever formalism for Kerr-comb generation in whispering-gallery-mode resonators," Phys. Rev. A 87, (2013).

18. Y. K. Chembo, D. V. Strekalov, and N. Yu, "Spectrum and Dynamics of Optical Frequency Combs Generated with Monolithic Whispering Gallery Mode Resonators," Phys. Rev. Lett. 104, (2010).

19. A. B. Matsko, A. A. Savchenkov, W. Liang, V. S. Ilchenko, D. Seidel, and L. Maleki, "Mode-locked Kerr frequency combs," Opt. Lett. 36, 2845 (2011).

20. T. Hansson, D. Modotto, and S. Wabnitz, "Dynamics of the modulational instability in microresonator frequency combs," Phys. Rev. A 88, 023819 (2013).

21. M. Haelterman, S. Trillo, and S. Wabnitz, "Dissipative modulation instability in a nonlinear dispersive ring cavity," Opt. Commun. 91, 401-407 (1992).

22. T. Hansson, D. Modotto, and S. Wabnitz, "On the numerical simulation of Kerr frequency combs using coupled mode equations," Opt. Commun. 312, 134-136 (2014).

23. C. Arlotti, O. Gauthier-Lafaye, A. Monmayrant, and S. Calvez, "Achromatic critically coupled racetrack resonators," J. Opt. Soc. Am. B 34, 2343 (2017). 
24. K. Ikeda, "Multiple-valued stationary state and its instability of the transmitted light by a ring cavity system," Opt. Commun. 30, 257261 (1979).

25. T. Hansson and S. Wabnitz, "Frequency comb generation beyond the Lugiato-Lefever equation: multi-stability and super cavity solitons," J. Opt. Soc. Am. B 32, 1259 (2015).

26. S. Balac and A. Fernandez, "SPIP : A computer program implementing the Interaction Picture method for simulation of light-wave propagation in optical fibre," Comput. Phys. Commun. 199, 139-152 (2016).

27. S. Balac, A. Fernandez, F. Mahé, F. Méhats, and R. Texier-Picard, "The Interaction Picture method for solving the generalized nonlinear Schrödinger equation in optics," ESAIM Math. Model. Numer. Anal. 50, 945-964 (2016).

28. J. Hult, "A Fourth-Order Runge-Kutta in the Interaction Picture Method for Simulating Supercontinuum Generation in Optical Fibers," J. Light. Technol. 25, 3770-3775 (2007).

29. D. J. Moss, R. Morandotti, A. L. Gaeta, and M. Lipson, "New CMOScompatible platforms based on silicon nitride and Hydex for nonlinear optics," Nat. Photonics 7, 597-607 (2013).

30. C. Bao and C. Yang, "Mode-pulling and phase-matching in broadband Kerr frequency comb generation," J. Opt. Soc. Am. B 31, 3074 (2014).

31. C. Bao, H. Taheri, L. Zhang, A. Matsko, Y. Yan, P. Liao, L. Maleki, and A. E. Willner, "High-order dispersion in Kerr comb oscillators," J. Opt. Soc. Am. B 34, 715 (2017).

32. T. Herr, K. Hartinger, J. Riemensberger, C. Wang, E. Gavartin, R. Holzwarth, M. L. Gorodetsky, and T. J. Kippenberg, "Universal formation dynamics and noise of Kerr frequency combs in microresonators," Nat. Photonics 6, 480-487 (2012).

33. A. Coillet, I. Balakireva, R. Henriet, K. Saleh, L. Larger, J. M. Dudley, C. R. Menyuk, and Y. K. Chembo, "Azimuthal Turing Patterns, Bright and Dark Cavity Solitons in Kerr Combs Generated With Whispering-Gallery-Mode Resonators," IEEE Photonics J. 5, 6100409-6100409 (2013).

34. M. Karpov, H. Guo, E. Lucas, A. Kordts, M. H. P. Pfeiffer, G. Lichachev, V. E. Lobanov, M. L. Gorodetsky, and T. J. Kippenberg, "Universal dynamics and controlled switching of dissipative Kerr solitons in optical microresonators," Nat. Phys. 13, 94-102 (2017).

35. Z. Kang, F. Li, J. Yuan, K. Nakkeeran, J. N. Kutz, Q. Wu, C. Yu, and P. K. A. Wai, "Deterministic generation of single soliton Kerr frequency comb in microresonators by a single shot pulsed trigger," Opt. Express 26, 18563 (2018).

36. A. B. Matsko, A. A. Savchenkov, V. S. Ilchenko, D. Seidel, and L. Maleki, "Hard and Soft Excitation Regimes of Kerr Frequency Combs," Phys. Rev. A 85, 023830 (2012).

37. Y. Okawachi, M. R. E. Lamont, K. Luke, D. O. Carvalho, M. Yu, M. Lipson, and A. L. Gaeta, "Bandwidth shaping of microresonatorbased frequency combs via dispersion engineering," Opt. Lett. 39, 3535 (2014).

38. S. Gundavarapu, G. M. Brodnik, M. Puckett, T. Huffman, D. Bose, R. Behunin, J. Wu, T. Qiu, C. Pinho, N. Chauhan, J. Nohava, P. T. Rakich, K. D. Nelson, M. Salit, and D. J. Blumenthal, "Sub-hertz fundamental linewidth photonic integrated Brillouin laser," Nat. Photonics 13, 60-67 (2019).

39. R. W. Boyd, Nonlinear Optics (Academic Press, 2008).

40. R. G. Smith, "Optical Power Handling Capacity of Low Loss Optical Fibers as Determined by Stimulated Raman and Brillouin Scattering," Appl. Opt. 11, 2489 (1972).

41. W. Liang, A. B. Matsko, A. A. Savchenkov, V. S. Ilchenko, D. Seidel, and L. Maleki, "Generation of Kerr Combs in MgF2 and CaF2 Microresonators," (2011). 